\title{
Los payasos de hospital en la impartición del grado de Medicina
}

\author{
Santiago Ambrosio, Angie Rosales, Antonio Masegosa, Javier Ariza
}

En el Campus de Bellvitge de la Facultad de Medicina de la Universitat de Barcelona se está desarrollando desde hace unos años una experiencia pedagógica enmarcada en el contexto de la adquisición de competencias comunicativas en la formación global del profesional de medicina.

La experiencia consiste en trasladar al aula la metodología de intervención de los payasos de hospital como profesionales del espectáculo y de la transmisión de positivismo y, a la vez, como conocedores del entorno hospitalario (pediátrico, geriátrico, psiquiátrico) desde un punto de vista distinto al del profesional de la salud. Se trata de aprovechar este bagaje para aportar herramientas complementarias al trato con el paciente desde una perspectiva estrictamente humana. Ha de decirse que dicha experiencia se puso en marcha por iniciativa de un estudiante que creía en ella y supo defenderla ante las instancias académicas del campus para su consideración como 'actividad de libre elección', ofertada a estudiantes de medicina y enfermería.

La facultad hizo suya la iniciativa a partir de los resultados de los tres primeros cursos. La experiencia encajaba en la adquisición de una competencia transversal contemplada en los nuevos planes de estudio como es la comunicación, en el sentido más amplio de comunicación médico-paciente, médicofamiliar, médico-enfermera, médico-médico, más allá del suministro de información y del intercambio de opiniones y conocimientos científicos.

La actividad se desarrolla actualmente como asignatura optativa de tres créditos ECTS, formando parte integral del plan de estudios del grado de Medicina que se imparte en la Universitat de Barcelona. Se estructura en ocho sesiones semanales de cuatro horas y un trabajo no presencial por parte del estudiante. La asignatura está coordinada por dos profesores de la Facultad de Medicina, vicedecano y secretario respectivamente, que se encargan de introducir la asignatura, supervisar sus contenidos en coherencia con otras asignaturas con com- petencias reconocidas de comunicación (psicología, semiología, oncología, asistencia primaria) y valorar los resultados. Los grupos de trabajo consisten en un máximo de 30 estudiantes de tercer curso (coincidiendo con el momento en el que entran en contacto con el paciente hospitalario por primera vez en nuestra facultad) y se dispone de un espacio de $150 \mathrm{~m}^{2}$ (un aula normalmente destinada a 100 estudiantes). En cada sesión participan dos profesionales de la asociación Pallapupas, cuya labor goza ya de un notable reconocimiento por la actividad desarrollada en importantes hospitales públicos de Cataluña a lo largo de los últimos 12 años, especialmente el Hospital Sant Joan de Déu de Barcelona [1].

Los objetivos y procedimientos toman como referencia el método Pallapupas y su modelo de intervención basado en los principios del 'teatro social' de Augusto Boal [2]. Se trata de entrar en el mundo de las emociones desde el juego, utilizando recursos teatrales para resolver conflictos. Se detectan las necesidades desde las experiencias o las creencias propias de cada individuo, para representarlas teatralmente y debatirlas. En ejercicios de role-playing se reproducen los papeles del médico, enfermero, celador, familiar y paciente, evidenciándose los prejuicios existentes en el mundo de la sanidad. Se ejercita la ardua tarea de reírse de uno mismo [3] y desarrollar el buen humor $[4,5]$, dando especial importancia al saber escuchar y al lenguaje no verbal.

Nos consta que experiencias de este tipo en el mundo de la educación médica se están intentando de forma muy puntual en la Universidad de Washington [6] y en la Universidad de Pennsylvania [7]. Más cerca de nosotros, la Universitat de Lleida ha llevado a cabo una actividad de libre elección sobre la risa como herramienta terapéutica [8]. Con todo, sólo en nuestra facultad se ha llegado a introducir en el plan de estudios en acción conjunta entre profesorado, profesional médico y payasos de hospital. Su principal objetivo es dar respuesta a la necesidad de formar al médico en aquellos aspectos más hu-
Departamento de Ciencias Fisiológicas II (S. Ambrosio); Departamento de Ciencias Clínicas (J. Ariza); Facultad de Medicina; Universitat de Barcelona. Servicio de Enfermedades Infecciosas; Hospital General Universitari de Bellvitge (J. Ariza). Asociación Pallapupas (A. Rosales, A. Masegosa). Barcelona, España.

Correspondencia: Dr. Santiago Ambrosio Viale. Departamento de Ciencias Fisiológicas II. Facultad de Medicina. Universitat de Barcelona-Idibell. Feixa Llarga, $\mathrm{s} / \mathrm{n}$. E-08907 L'Hospitalet de Llobregat (Barcelona).

E-mail: sambrosio@ub.edu (C) 2013 FEM 
manos de la comprensión, tolerancia y relaciones humanas, desde la vertiente del sentido del humor. Los resultados obtenidos hasta la fecha son muy satisfactorios, en lo referente a la aceptación y apreciación entre el alumnado y el profesorado, y la experiencia resulta lo bastante innovadora y prometedora como para darla a conocer.

Bibliografía

1. Pallapupas. URL: http://www.pallapupas.org/es/pallapupas/ portadaquisom.html.

2. Boal A. Teatro del oprimido: juegos para actores y no actores. Barcelona: Alba Editorial; 2002.
3. Wild B, Rodden FA, Grodd W, Ruch W, Neural correlates of laughter and humor. Brain 2003; 126: 2121-38.

4. Penson RT, Partridge RA, Rudd P, Seiden MV, Nelson JE, Chabner BA, et al. Laughter: the best medicine? Oncologist 2005; 10: 651-60.

5. Arias M, Neurología de la risa y el humor: risa y llanto patológicos. Rev Neurol 2011; 53: 415-21.

6. Abendschein J, Abendschein D. Humor in medical school -from classroom to bedside. Association for Applied and Therapeutic Humor Annual Conference. March 1-2, 2003.

7. King E. Society for the Arts in Healthcare. URL: http:// www1074.ssldomain.com/thesah/cs/person_detail. cfm?person id $=1958$.

8. Viana A. La risa y su función terapéutica. Una experiencia docente. URL: http://www.filcat.udl.cat/unic/risa terapeutica.pdf. 